\title{
Development of a novel method for visualizing restricted diffusion using subtraction of apparent diffusion coefficient values
}

\author{
YUUKI YOSHIMURA $^{1,2}$, MASAHIRO KURODA ${ }^{1}$, IRFAN SUGIANTO ${ }^{3,4}$, ABDULLAH KHASAWNEH $^{3}$, \\ BABATUNDE O. BAMGBOSE ${ }^{3}$, KENTARO HAMADA $^{1}$, MAJD BARHAM $^{3}$, NOUHA TEKIKI $^{3}$, AKIRA KUROZUMI $^{5}$, \\ TOSHI MATSUSHITA ${ }^{5}$, SEIICHIRO OHNO ${ }^{5}$, SUSUMU KANAZAWA ${ }^{6}$ and JUNICHI ASAUMI ${ }^{3}$ \\ ${ }^{1}$ Department of Radiological Technology, Graduate School of Health Sciences, Okayama University, Okayama 7008558; \\ ${ }^{2}$ Department of Radiology Diagnosis, Okayama Saiseikai General Hospital, Okayama 7008511; ${ }^{3}$ Department of Oral and \\ Maxillofacial Radiology, Okayama University Graduate School of Medicine, Dentistry and Pharmaceutical Sciences, \\ Okayama 7008558, Japan; ${ }^{4}$ Department of Oral Radiology, Faculty of Dentistry, Hasanuddin University, \\ Makassar 90245, Indonesia; ${ }^{5}$ Central Division of Radiology, Okayama University Hospital; ${ }^{6}$ Department of Radiology, \\ Okayama University Graduate School of Medicine, Dentistry and Pharmaceutical Sciences, Okayama 7008558, Japan
}

Received February 15, 2019; Accepted July 2, 2019

DOI: $10.3892 / \mathrm{mmr} .2019 .10523$

\begin{abstract}
In order to visualize restricted diffusion, the present study developed a novel method called 'apparent diffusion coefficient (ADC) subtraction method (ASM)' and compared it with diffusion kurtosis imaging (DKI). The diffusion-weighted images of physiological saline, in addtion to bio-phatoms of low cell density and the highest cell density were obtained using two sequences with different effective diffusion times. Then, the calculated ADC values were subtracted. The mean values and standard deviations (SD) of the ADC values of physiological saline, low cell density and the highest cell density phantoms were $2.95 \pm 0.08 \times 10^{-3}, 1.90 \pm 0.35 \times 10^{-3}$ and $0.79 \pm 0.05 \times 10^{-3} \mathrm{~mm}^{2} / \mathrm{sec}$, respectively. The mean kurtosis values and SD of DKI were $0.04 \pm 0.01,0.44 \pm 0.13$ and $1.27 \pm 0.03$, respectively. The ASM and SD values were $0.25 \pm 0.20 \times 10^{4}$, $0.51 \pm 0.41 \times 10^{4}$ and $4.80 \pm 4.51 \times 10^{4}\left(\mathrm{sec} / \mathrm{mm}^{2}\right)^{2}$, respectively. Using bio-phantoms, the present study demonstrated that DKI exhibits restricted diffusion in the extracellular space. Similarly, ASM may reflect the extent of restricted diffusion in the extracellular space.
\end{abstract}

\section{Introduction}

Diffusion weighted images (DWIs) have been reported useful for early diagnosis of tumors and cerebrovascular disorders

Correspondence to: Professor Masahiro Kuroda, Department of Radiological Technology, Graduate School of Health Sciences, Okayama University, 2-5-1 Shikata-cho, Okayama 7008558, Japan E-mail: kurodamd@cc.okayama-u.ac.jp

Key words: magnetic resonance imaging, apparent diffusion coefficient, diffusion kurtosis imaging, subtraction, restricted diffusion, bio-phantom, cell such as cerebral infarction in clinical practice for magnetic resonance imaging (MRI) (1-3). The apparent diffusion coefficient (ADC) map calculated from DWIs is also used clinically, reflecting both free and restricted diffusion. Free diffusion occurs in the absence of a physical barrier and represents the normal distribution of water molecules spreading during a certain time. Restricted diffusion occurs with barriers such as membranes and multiple compartments in living organisms (4).

Recent techniques such as diffusion kurtosis imaging (DKI) $(5,6)$, which analyze the movement of water molecules in restricted diffusion, have been reported. DKI shows restricted diffusion as an index of kurtosis, indicating the degree of deviation from the normal distribution. Recent clinical research indicates that DKI is useful to diagnose acute stage cerebral infarction, glioma, Alzheimer's disease, Parkinson's disease, attention deficit hyperactivity disorder, multiple sclerosis, temporal lobe epilepsy, traumatic brain injury and spinal cord lesions (7-20). Among restricted diffusion imaging techniques, DKI has advantages such as high specificity for restricted diffusion; a small number of b-values, which results in a relatively short imaging time; and quantitative capability. However, it has disadvantages such as the difficulty to understand intuitively the value of the kurtosis, due to the lack of assumption of the biophysical model; the variation in the kurtosis depending on the range of the b-value; and the requirement for specialized software for DKI. For the above reasons, DKI has not been used in routine clinical practice.

The present study developed a novel method to visualize restricted diffusion differently from DKI. Two ADC values with different diffusion times were used, and the difference between them was calculated. This method was referred to as 'apparent diffusion coefficient (ADC) subtraction method (ASM)'. The purpose of the present study was to compare ASM and DKI in order to examine whether ASM can reveal restricted diffusion using a cell-containing bio-phantom that was developed by our group. 


\section{Materials and methods}

Phantom container. A microcuvette (halbmikro $1.5 \mathrm{ml}$; Greiner Labortechnik Manufacturing Ltd., Greiner, Germany) was installed in a phantom container that had an outer diameter of $9.5 \mathrm{~cm}$ in length, $14 \mathrm{~cm}$ in width and $7 \mathrm{~cm}$ in height (21). The interior of the container was filled with physiological saline $(0.9 \% \mathrm{NaCl})$.

Bio-phantom. As bio-phantom, Jurkat cells were used, which were purchased from Bio Resource Center (Tsukuba, Japan). For cell culture, 10\% fetal bovine serum (Filtron Pty Ltd., Victoria, Australia) and $1 \%$ penicillin-streptomycin-neomycin (Gibco; Thermo Fisher Scientific, Inc., Waltham, MA, USA) were added to RPMI-1640 medium (pH 7.4; Gibco; Thermo Fisher Scientific, Inc.). The incubation was carried out at $37^{\circ} \mathrm{C}$ with $5 \% \mathrm{CO}_{2}$. The number of cells with a diameter $>8 \mu \mathrm{m}$ was counted with an electric cell counter (Coulter Electronics Ltd., Luton, UK) prior to bio-phantom preparation, since the diameter of the majority of Jurkat cells is $>8 \mu \mathrm{m}$, with the mean diameter being $9.6 \mu \mathrm{m}$ (22). The Jurkat cells were encapsulated into bio-phantoms as previously described (22). Briefly, upon measuring the cell number, the cell solution was concentrated to $\sim 0.89 \mathrm{ml}$, placed in a micro-cuvette (halbmikro $1.5 \mathrm{ml}$; Greiner Labortechnik Manufacturing Ltd.) and centrifuged at $161 \mathrm{x}$ g for $5 \mathrm{~min}$. Next, the supernatant was removed and the cell density was adjusted to $\sim 1-8 \times 10^{8}$ cells $/ \mathrm{ml}$. Upon treatment, the cells were enclosed in gellan gum (P-8169; Sigma-Aldrich; Merck KGaA, Darmstadt, Germany). Two types of bio-phantom were prepared. One was a pellet-like high cell density phantom, and the other was a low cell density phantom fixed with gellan gum. Each bio-phantom was enclosed in a phantom container.

MRI device, image analysis software and statistical analysis software. A 3.0T MRI system (MAGNETOM Prisma VE11C; Siemens AG, Munich, Germany) was used, which had a 20-channel head/neck coil. The image analysis software used was Image $\mathbf{J}$ (National Institutes of Health, Bethesda, MD, USA) (23). The DKI image analysis software used was diffusional kurtosis estimator (DKE) version 2.6 (24). Statcel4, which is an add-in of Excel (Microsoft Corporation, Redmond, WA, USA) was used for statistical analysis.

Bio-phantom heating device. A phantom container was installed in a self-constructed bio-phantom heating device, which was formed of ethylene-vinyl acetate copolymer and was connected to a circulating thermostatic chamber (Thermo-Mate BF-41; Yamato Scientific Co., Ltd., Tokyo, Japan). The temperature of the bio-phantom was adjusted to $\sim 37^{\circ} \mathrm{C}$, similarly to human body temperature.

Temperature measurement during MRI. For real-time phantom temperature measurements, an optical fiber thermometer (Fluoroptic ${ }^{\mathrm{TM}}$ m3300, Luxtron Co., Santa Clara, CA, USA) was installed in the micro-cuvette during MRI.

Imaging conditions. Table I shows the imaging conditions of DKI and ASM. In DKI, single shot-echo planar imaging (SS-EPI) (25) was used in three sequences of DKI-1, DKI-2 and DKI-3. In ASM, two types of readout segmentation of long variable echo-trains (RESOLVE) (26) sequences were used, namely RESOLVE-basic and RESOLVE-modify. Two types of DWI were obtained for ASM by changing the number of $b$-values. For RESOLVE-basic, the b-values were set to 3 points: 0,500 and $1,000 \mathrm{sec} / \mathrm{mm}^{2}$. For RESOLVE-modify, the b-values were set to 4 points: $0,500,1,000$ and $10,000 \mathrm{sec} / \mathrm{mm}^{2}$ (Table I). Since the number of b-values was different, the $\delta$ [motion probing gradient (MPG) pulse duration] and $\Delta$ (MPG pulse spacing) of both sequences changed. In the formula used to calculate b-values (Equation 1), ' $\Delta-\delta / 3$ ' is called the effective diffusion time and represents the time during which diffusion phenomena are observed.

$$
b=\gamma^{2} G^{2} \delta^{2}(\Delta-\delta / 3)
$$

(Equation 1)

In the above formula, $\gamma$ is the gyromagnetic ratio of protons and $\mathrm{G}$ is the gradient magnetic field strength. The effective diffusion time of RESOLVE-basic and RESOLVE-modify were 39.3 and $46.0 \mathrm{msec}$, respectively. The extension of the effective diffusion time has an upper limit. The effective diffusion time of this modification sequence is elongated until this limit. Imaging of both DKI and ASM was performed 5 times for the high cell density phantom and 9 times for the low cell density phantom.

Image processing of DKI. DKI image analysis software DKE version 2.6 is published on the website of the Medical University of South Carolina (http://academicdepartments. musc.edu/cbi//dki). The DWIs obtained by imaging of DKI-1, DKI-2 and DKI-3 were processed with DKE to prepare a mean kurtosis (MK) image (Equation 2), which is a mean in the spatial direction. By interpolated processing, the voxel size of the MK image becomes 1.0x1.0x1.0 mm.

$$
\mathrm{S}=\mathrm{S}_{0}{ }^{*} \exp \left(-\mathrm{b}^{*} \mathrm{ADC}+\mathrm{b}^{2 *} \mathrm{ADC}{ }^{2 *} \mathrm{MK} / 6\right)
$$

(Equation 2)

In the above formula, $\mathrm{S}$ is signal intensity and $\mathrm{S}_{0}$ is the signal intensity when the $b$-value is $0 \mathrm{sec} / \mathrm{mm}^{2}$. The $b$-values used are shown in Table I.

Image processing of $A S M$. The $\mathrm{ADC}$ values $\left(\mathrm{ADC}_{\mathrm{b}}\right.$ and $\left.\mathrm{ADC}_{\mathrm{m}}\right)$ were calculated for RESOLVE-basic using the 3 b-values: 0,500 and $1,000 \mathrm{sec} / \mathrm{mm}^{2}$, and for RESOLVE-modify using the above $3 \mathrm{~b}$-values from 0 to 1,000 without $10,000 \mathrm{sec} / \mathrm{mm}^{2}$. The formula used to calculate ASM is shown in Equation 3. As the variation of $\mathrm{ADC}$ values increases when $\mathrm{ADC}$ values are high, the absolute difference between $\mathrm{ADC}$ values $\left(\mathrm{ADC}_{\mathrm{b}}\right.$ and $\mathrm{ADC}_{\mathrm{m}}$ ) is divided by $\mathrm{ADC}_{\mathrm{b}}$ value three times in $\mathrm{ASM}$ to adjust the variation of $\mathrm{ADC}$ values.

$$
\mathrm{ASM}=\mid \mathrm{ADC}_{\mathrm{b}}-\mathrm{ADC}_{\mathrm{m}} \mathrm{l} /\left(\mathrm{ADC}_{\mathrm{b}}\right)^{3}
$$

(Equation 3)

Image evaluation. Regarding the MK image, the MK values were determined from three regions of interest (ROI) of $1 \mathrm{x} 4$ pixels in the cell part inside the bio-phantom, and from ROIs of the same size in 6 areas of the physiological saline portion inside the phantom container. With regard to ASM, the signal intensity was determined from four ROIs of $3 \times 3$ pixels selected in the cell part of the bio-phantom. For DWI, each 
Table I. Imaging conditions of diffusion kurtosis imaging and ASM.

\begin{tabular}{|c|c|c|c|c|c|}
\hline \multirow[b]{2}{*}{ Parameters } & \multicolumn{2}{|c|}{ ASM } & \multicolumn{3}{|c|}{ DKI } \\
\hline & RESOLVE-basic & RESOLVE-modify & DKI-1 & DKI-2 & DKI-3 \\
\hline TR (msec) & 8,000 & 8,000 & 6,000 & 6,000 & 6,000 \\
\hline TE (msec) & 86 & 106 & 75 & 75 & 75 \\
\hline $\mathrm{ES}(\mathrm{msec})$ & 0.56 & 0.56 & 0.93 & 0.93 & 0.93 \\
\hline FOV (mm) & 120 & 120 & 120 & 120 & 120 \\
\hline Matrix & $224 \times 224$ & $224 \times 224$ & $82 \times 82$ & $82 \times 82$ & $82 \times 82$ \\
\hline BW (Hz/pixel) & 399 & 399 & 1,220 & 1,220 & 1,220 \\
\hline Averages & 2 & 2 & 1 & 1 & 9 \\
\hline Segments & 7 & 7 & 1 & 1 & 1 \\
\hline Slice thickness (mm) & 5 & 5 & 5 & 5 & 5 \\
\hline Slice number & 1 & 1 & 5 & 5 & 5 \\
\hline Phase direction & $\mathrm{AP}$ & AP & $\mathrm{AP}$ & AP & AP \\
\hline$\delta(\mathrm{msec})$ & 5.6 & 15.6 & 13.8 & 13.8 & - \\
\hline$\Delta(\mathrm{msec})$ & 41.2 & 51.2 & 33.5 & 33.5 & - \\
\hline Diffusion time (msec) & 39.3 & 46.0 & 28.9 & 28.9 & - \\
\hline b-value $\left(\mathrm{sec} / \mathrm{mm}^{2}\right)$ & $0,500,1,000$ & $0,500,1,000,10,000$ & $0,500,1,000$ & $0,500,1,000$ & 0 \\
\hline Diffusion direction & 3 & 3 & 30 & 30 & - \\
\hline Imaging time (min:sec) & $13: 28$ & $19: 06$ & $6: 24$ & $6: 24$ & $1: 12$ \\
\hline
\end{tabular}

ASM, apparent diffusion coefficient subtraction method; DKI, diffusion kurtosis imaging; RESOLVE, readout segmentation of long variable echo-trains; TR, repetition time; TE, echo time; ES, echo space; FOV, field of view; BW, band width; AP, antero-posterior; $\delta$, motion probing gradient (MPG) pulse duration; $\Delta$, MPG pulse spacing.

b-value was obtained from the imaging of RESOLVE-basic and RESOLVE-modify. The signal intensity was also determined from 8 same-sized ROIs located in the physiological saline portion of the phantom container. Each signal intensity value was logarithmically transformed. Then, the ADC value for each ROI was calculated from the inverse of the slope and the ASM value was calculated using Equation 3.

Regarding the $\mathrm{ADC}_{\mathrm{b}}, \mathrm{MK}$ and $\mathrm{ASM}$ values, a multiple comparison test using the Steel-Dwass method was performed at a significance level of $5 \%$ between saline, low cell density phantom and the highest cell density phantom.

The total number of ROIs used for the calculation of the ADC and ASM values were 112 for physiological saline, 36 for low cell density phantom and 20 for the highest cell density phantom. The values used for the calculation of MK values were 84 for normal saline, 27 for low cell density phantom and 15 for the highest cell density phantom.

\section{Results}

The mean temperature and SD inside the bio-phantom during imaging were $37.2 \pm 0.7^{\circ} \mathrm{C}$. The cell densities of the low cell density phantom and the highest cell density phantom were $1.21 \times 10^{8}$ and $7.41 \times 10^{8}$ cells $/ \mathrm{ml}$, respectively. The mean $\mathrm{ADC}_{\mathrm{b}}$ and SD values of physiological saline, low cell density phantom and the highest cell density phantom were $2.95 \pm 0.08 \times 10^{-3}$, $1.90 \pm 0.35 \times 10^{-3}$ and $0.79 \pm 0.05 \times 10^{-3} \mathrm{~mm}^{2} / \mathrm{sec}$, respectively (Fig. 1). As the cell density increased, the $\mathrm{ADC}_{\mathrm{b}}$ decreased. A significant difference was observed using the Steel-Dwass

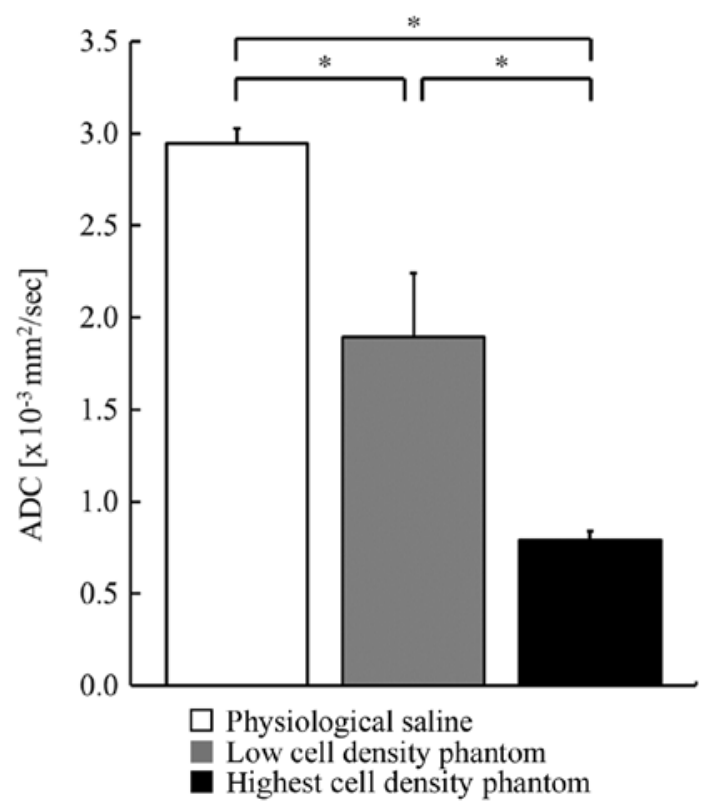

Figure 1. ADC values using RESOLVE-basic. White, gray and black columns indicate physiological saline, low cell density phantom and the highest cell density phantom, respectively. ${ }^{*} \mathrm{P}<0.05$. RESOLVE, readout segmentation of long variable echo-trains. ADC, apparent diffusion coefficient.

method among the 3 groups. The mean MK and SD values of physiological saline, low cell density phantom and the highest cell density phantom were $0.04 \pm 0.01,0.44 \pm 0.13$ and $1.27 \pm 0.03$, respectively (Fig. 2). The mean ASM and SD values of 


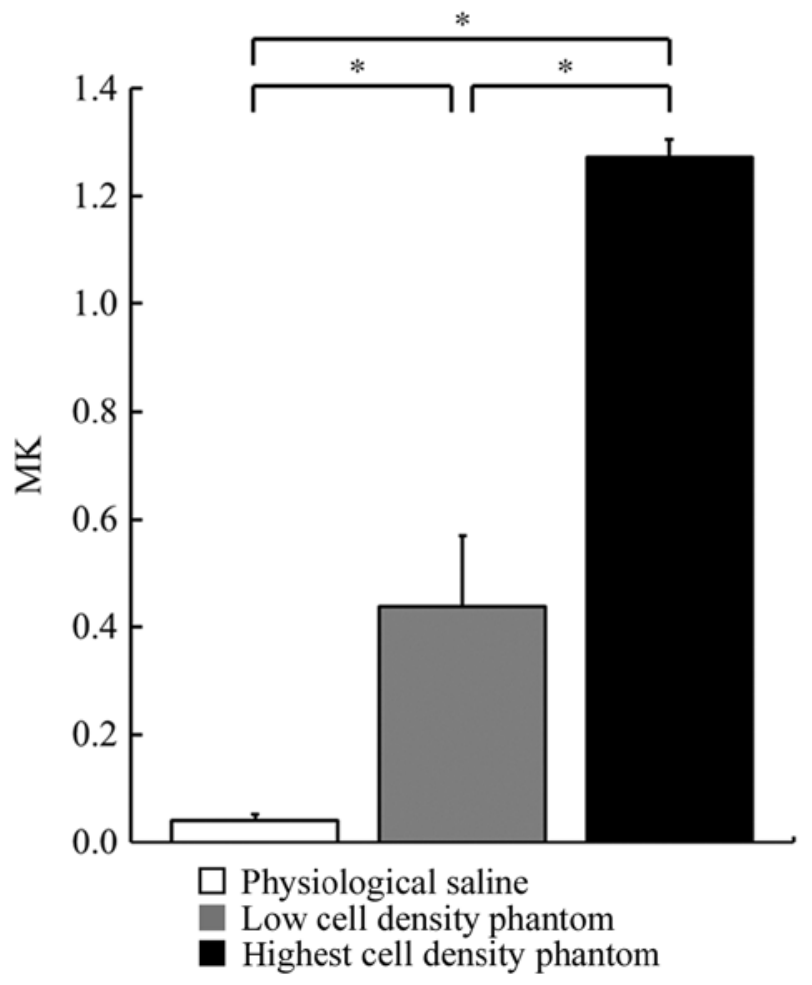

Figure 2. Mean kurtosis values. White, gray and black columns indicate physiological saline, low cell density phantom and the highest cell density phantom, respectively. ${ }^{*} \mathrm{P}<0.05$.

physiological saline, low cell density phantom and the highest cell density phantom were $0.25 \pm 0.20 \times 10^{4}, 0.51 \pm 0.41 \times 10^{4}$ and $4.80 \pm 4.51 \times 10^{4}\left(\mathrm{sec} / \mathrm{mm}^{2}\right)^{2}$, respectively (Fig. 3). In contrast to the $\mathrm{ADC}_{\mathrm{b}}$, the MK and $\mathrm{ASM}$ values increased as the cell density increased. Significant differences were observed among the 3 groups for both MK and ASM values.

\section{Discussion}

In the present study, a novel method named ASM was developed and its usefulness was demonstrated experimentally.

DWI is widely used clinically. There are two types of diffusion: Free and restricted, and both are represented by the ADC value. In recent years, imaging techniques such as DKI, which expresses restricted diffusion, have appeared and have been reported to be useful in the clinic. However, the method of imaging restricted diffusion is limited. In the present study, a novel method named ASM was developed and its usefulness was demonstrated experimentally. DKI is an imaging technique that quantitatively reveals how water molecules deviate from free diffusion. DKI has been reported to have more potential to evaluate the actual microstructure in vivo than an ADC map $(5,6)$. Among restricted diffusion imaging techniques, DKI has advantages such as high specificity for restricted diffusion; a small number of b-values, which results in a relatively short imaging time; and quantitative capability. Greater the b-values, the stronger the diffusion weighting, the higher the contrast in pathogenic tissues. In the present study, DKI, which is a diffusion analysis method of non-normal distributions, was considered the standard to evaluate restricted diffusion and was compared with ASM.

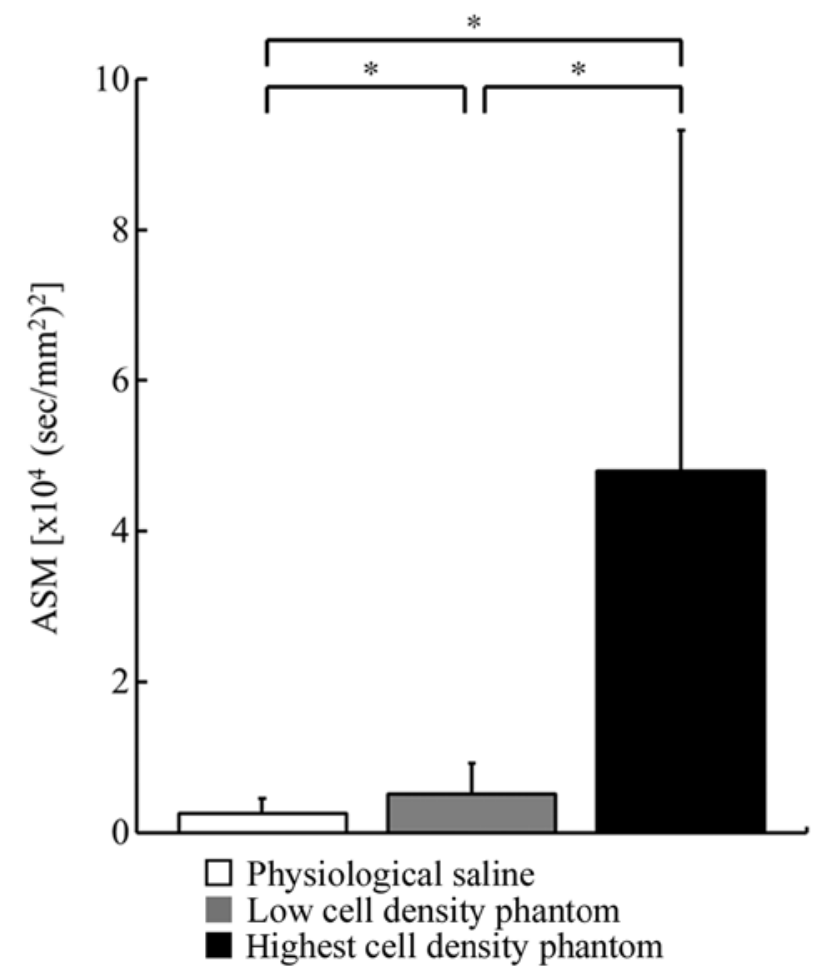

Figure 3. ASM values. White, gray and black columns indicate physiological saline, low cell density phantom and the highest cell density phantom, respectively. ${ }^{*} \mathrm{P}<0.05$. ASM, apparent diffusion coefficient subtraction method.

Numerous clinical studies using DKI have been reported. Hempel et al (27) observed a strong correlation between the grade of glioma and the MK value, stating that the higher the grade, the higher the MK value. Qi et al (28) also reported that the MK value increases as the grade of glioma and cell density increase. Wu et al (29) stated that the ADC value increases, while the MK value decreases, as a result of chemotherapy in cervical non-Hodgkin lymphoma. Wang et al (30) reported that the ADC value is low, while the MK value is high, in bladder tumors compared with bladder inflammation. Barrett et al (31) also stated that ADC values are low while MK values high in prostate cancer compared with normal prostate tissue. These results were similar to those of our study using bio-phantoms.

In the present study, the distance of the extracellular space was calculated by Kepler conjecture (32), with cell densities of the low cell density phantom and the highest cell density phantom of $1.21 \times 10^{8}$ and $7.41 \times 10^{8}$ cells $/ \mathrm{ml}$, respectively. The Kepler conjecture is a mathematical theorem about sphere packing in three-dimensional Euclidean space, which states that the maximum volume of closely-packed equally-sized spheres is $\sim 74.05 \%$ of the total volume of the Euclidean space. The total volume of the spheres in $1 \mathrm{ml}$ is $0.7405 \mathrm{ml}$, which is $74.05 \%$. Assuming that the cell is a sphere, the volume of one sphere can be determined by dividing the total volume of the spheres by the cell density. The radius of the sphere is calculated from the volume of the sphere. Since the cells are not closely packed in $1 \mathrm{ml}$, the calculated radius of the sphere is larger than the actual cell radius. Doubling the calculated radius of the sphere indicates the distance between cell centers. The cell diameter minus this distance between cell centers is 
A Low cell density phantom

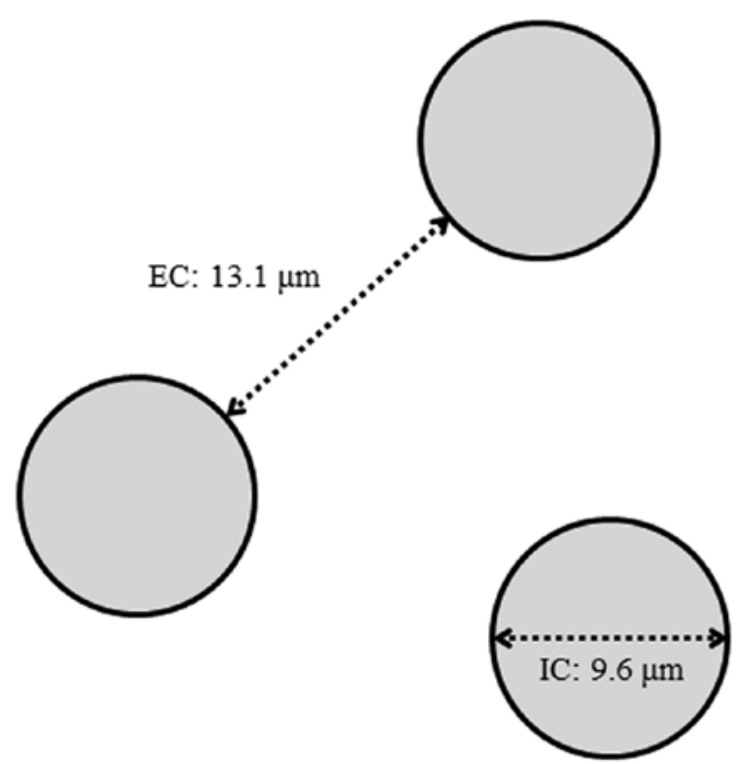

B Highest cell density phantom

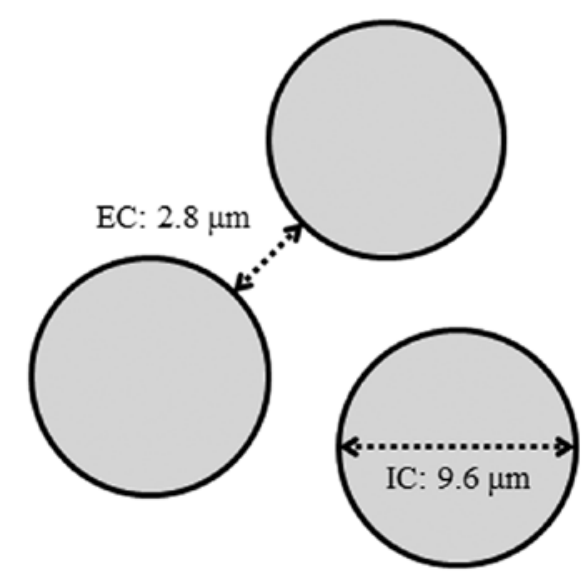

Observation range

ASM (present study): $\quad 1.0 \mu \mathrm{m}$

$\mathrm{ADC}_{\mathrm{b}}: \quad 12.3 \mu \mathrm{m}$

$\mathrm{ADC}_{\mathrm{m}}: \quad 13.3 \mu \mathrm{m}$

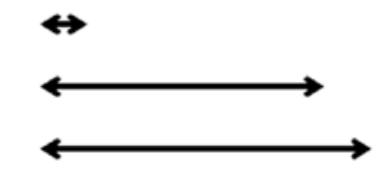

Figure 4. Correlation between the size of intracellular and extracellular spaces and the diffusion observation range using each method. (A) Low cell density phantom. (B) The highest cell density phantom. IC, intracellular space; EC, extracellular space; $\mathrm{ADC}_{\mathrm{b}}$, the apparent diffusion coefficient value using RESOLVE-basic; $\mathrm{ADC}_{\mathrm{m}}$, apparent diffusion coefficient value using RESOLVE-modify. RESOLVE, readout segmentation of long variable echo-trains.

defined as the distance between cell membranes (extracellular space) to evaluate the volume of the extracellular space. Since the cell diameter used in the present study is $9.6 \mu \mathrm{m}(22)$, the distance of the extracellular space of the low cell density phantom is $13.1 \mu \mathrm{m}$, while that of the maximum cell density phantom is $2.8 \mu \mathrm{m}$ (Fig. 4). The size of the intracellular space is the cell diameter, i.e. $9.6 \mu \mathrm{m}$.

The observation range of the diffusion phenomenon was calculated from the used effective diffusion time. This range changes according to the effective diffusion time. The subtraction of two ADC values via ASM, obtained using different diffusion times, may enable to observe diffusion phenomena in a narrow space. Although the ADC value itself may reflect both free and restricted diffusion, ASM may obtain information on restricted diffusion between cell membranes. The effective diffusion times of RESOLVE-basic and RESOLVE-modify are 39.3 and $46.0 \mathrm{msec}$, respectively. The Stokes-Einstein equation (33) indicates that water diffusion at $37^{\circ} \mathrm{C}$ is $3.0 \times 10^{-3} \mathrm{~mm}^{2} / \mathrm{sec}$. The range of water diffusion can be obtained by multiplying the ADC value of water, which is $3.0 \times 10^{-3} \mathrm{~mm}^{2} / \mathrm{sec}$ at $37^{\circ} \mathrm{C}$, by the effective diffusion time. Then, the diameter of the range of water diffusion was calculated to be 12.3 and $13.3 \mu \mathrm{m}$ for $\mathrm{ADC}_{\mathrm{b}}$ using RESOLVE-basic and $\mathrm{ADC}_{\mathrm{m}}$ using RESOLVE-modify, respectively. ASM may represent the difference between the two diameters, a range of $1.0 \mu \mathrm{m}$ (Fig. 4).
In our results, the higher the cell density and the narrower the extracellular space, the lower was the ADC value, and conversely, both MKand ASMvalues increased.Conventionally, DKI is expected to be able to image restricted diffusion, and our data also support that DKI reflects restricted diffusion in the extracellular space. Similarly, ASM may express the extent of restricted diffusion in the extracellular space.

In the present study, the imaging time of ASM (32.5 min in total) was longer than that of DKI (14 min in total). The image quality of DKI is lower than that of ASM. RESOLVE, used in $\mathrm{ASM}$, is reported to improve image quality without distortion and make ADC values accurate (21). The reason for the length of the imaging time of current ASM is the high resolution and the high number of averages and segments to improve the image quality of ASM. If the image quality of ASM is set to be the same as that of DKI, the imaging time of ASM decreases to $\sim 6$ min, which is shorter than that of DKI. In the future, it is necessary to develop an ASM sequence for clinical research that shortens the imaging time while maintaining the image quality.

In ASM using RESOLVE-basic and RESOLVE-modify, the present study did not explore the effect of varying each effective diffusion time. If each effective diffusion time used in ASM differs, the degree of restricted diffusion may change. However, the 3.0T devices commonly used clinically have limitations in regard to changing the range of diffusion time remarkably. 
In conclusion, using bio-phantoms, the present study clarified that DKI mainly reflects restricted diffusion in the extracellular space. Similarly, ASM may reflect the extent of restricted diffusion in the extracellular space. Future clinical studies are expected to demonstrate the potential of ASM as a useful tool for clinical imaging such as ADC maps.

\section{Acknowledgements}

Not applicable.

\section{Funding}

The present study was partially supported by Grants-in-Aid for Scientific Research (grant nos. C22591335, 15K09924 and 19K0809801) from the Ministry of Health, Labour and Welfare of Japan.

\section{Availability of data and materials}

The datasets used and/or analyzed during the current study are available from the corresponding author on reasonable request.

\section{Authors' contributions}

YY and MK conceived and designed the study, processed the data and wrote the article. YY, MK, IS, AK, BOB, KH, MB, NT, AK, TM, SO, SK and JA performed the experiments. IS, $\mathrm{AK}, \mathrm{KH}, \mathrm{MB}$ and NT edited the article. All authors read and approved the final version of the manuscript.

\section{Ethics approval and consent to participate}

Not applicable.

\section{Patient consent for publication}

Not applicable.

\section{Competing interests}

The authors declare that they have no competing interests.

\section{References}

1. Bammer R, Keeling SL, Augustin M, Pruessmann KP, Wolf R, Stollberger R, Hartung HP and Fazekas F: Improved diffusion-weight single-shot echo-planar imaging (EPI) in stroke using sensitivity encoding (SENSE). Magn Reson Med 46: 548-554, 2001.

2. Takahara T, Imai Y, Yamashita T, Yasuda S, Nasu S and Van Cauteren M: Diffusion weighted whole body imaging with background body signal suppression (DWIBS): Technical improvement using free breathing, STIR and high resolution 3D display. Radiat Med 22: 275-282, 2004.

3. Nasu K, Kuroki Y, Nawano S, Kuroki S, Tsukamoto T, Yamamoto S, Motoori K and Ueda T: Hepatic metastases: Diffusion-weighted sensitivity-encoding versus SPIO-enhanced MR imaging. Radiology 239: 122-130, 2006.

4. Hori M, Fukunaga I, Masutani Y, Taoka T, Kamagata K, Suzuki Y and Aoki S: Visualizing non-Gaussian diffusion: Clinical application of q-space imaging and diffusional kurtosis imaging of the brain and spine. Magn Reson Med Sci 11: 221-33, 2012.
5. Jensen JH, Helpern JA, Ramani A, Lu H and Kaczynski K: Diffusional kurtosis imaging: The quantification of non-gaussian water diffusion by means of magnetic resonance imaging. Magn Reson Med 53: 1432-1440, 2005.

6. Jensen JH and Helpern JA: MRI Quantification of non-Gaussian water diffusion by kurtosis analysis. NMR Biomed 23: 698-710, 2010.

7. Jensen JH, Falangola MF, Hu C, Tabesh A, Rapalino O, Lo C and Helpern JA: Preliminary observations of increased diffusional kurtosis in human brain following recent cerebral infarction. NMR Biomed 24: 452-457, 2011.

8. Taoka T, Fujioka M, Sakamoto M, Miyasaka T, Akashi T, Ochi T, Hori S, Uchikoshi M, Xu J and Kichikawa K: Time course of axial and radial diffusion kurtosis of white matter infarctions: Period of pseudonormalization. Am J Neuroradiol 35: 1509-1514, 2014.

9. Raab P, Hattingen E, Franz K, Zanella FE and Lanfermann H: Cerebral gliomas: Diffusional kurtosis imaging analysis of microstructural differences. Radiology 254: 876-881, 2010.

10. Van Cauter S, Veraart J, Sijbers J, Peeters RR, Himmelreich U, De Keyzer F, Van Gool SW, Van CalenberghF, De Vleeschouwer S, Van Hecke W and Sunaert S: Gliomas: Diffusion kurtosis MR imaging in grading. Radiology 263: 492-501, 2012.

11. Gong NJ, Wong CS, Chan CC, Leung LM and Chu YC: Correlations between microstructural alterations and severity of cognitive deficiency in Alzheimer's disease and mild cognitive impairment: A diffusional kurtosis imaging study. Magn Reson Imaging 31: 688-694, 2013

12. Wang JJ, Lin WY, Lu CS, Weng YH, Ng SH, Wang CH, Liu HL, Hsieh RH, Wan YL and Wai YY: Parkinson disease: Diagnostic utility of diffusion kurtosis imaging. Radiology 261: 210-217, 2011.

13. Kamagata K, Tomiyama H, Motoi Y, Kano M, Abe O, Ito K, Shimoji K, Suzuki M, Hori M, Nakanishi A, et al: Diffusional kurtosis imaging of cingulate fibers in Parkinson disease: Comparison with conventional diffusion tensor imaging. Magn Reson Imaging 31: 1501-1506, 2013.

14. Kamagata K, Tomiyama H, Hatano T, Motoi Y, Abe O, Shimoji K, Kamiya K, Suzuki M, Hori M, Yoshida M, et al: A preliminary diffusional kurtosis imaging study of Parkinson disease: Comparison with conventional diffusion tensor imaging. Neuroradiology 56: 251-258, 2014.

15. Adisetiyo V, Tabesh A, Di Martino A, Falangola MF, CastellanosFX,JensenJHandHelpernJA:Attention-deficit/hyperactivity disorder without comorbidity is associated with distinct atypical patterns of cerebral microstructural development. Hum Brain Mapp 35: 2148-2162, 2014.

16. Yoshida M, Hori M, Yokoyama K, Fukunaga I, Suzuki M, Kamagata K, Shimoji K, Nakanishi A, Hattori N, Masutani Y and Aoki S: Diffusional kurtosis imaging of normal-appearing white matter in multiple sclerosis: Preliminary clinical experience. Jpn J Radiol 31: 50-55, 2013.

17. Gao Y, Zhang Y, Wong CS, Wu PM, Zhang Z, Gao J, Qiu D and Huang B: Diffusion abnormalities in temporal lobes of children with temporal lobe epilepsy: A preliminary diffusional kurtosis imaging study and comparison with diffusion tensor imaging. NMR Biomed 25: 1369-1377, 2012.

18. Grossman EJ, Jensen JH, Babb JS, Chen Q, Tabesh A, Fieremans E, Xia D, Inglese $M$ and Grossman RI: Cognitive impairment in mild traumatic brain injury: A longitudinal diffusional kurtosis and perfusion imaging study. Am J Neuroradiol 34: 951-957, 2013.

19. Raz E, Bester M, Sigmund EE, Tabesh A, Babb JS, Jaggi H, Helpern J, Mitnick RJ and Inglese M: A better characterization of spinal cord damage in multiple sclerosis: A diffusional kurtosis imaging study. AJNR Am J Neuroradiol 34: 1846-1852, 2013.

20. Hori M, Tsutsumi S, Yasumoto Y, Ito M, Suzuki M, Tanaka FS, Kyogoku S, Nakamura M, Tabuchi T, Fukunaga I, et al: Cervical spondylosis: Evaluation of microstructural changes in spinal cord white matter and gray matter by diffusional kurtosis imaging. Magn Reson Imaging 32: 428-432, 2014.

21. Yoshimura Y, Kuroda M, Sugianto I, Bamgbose BO, Miyahara K, Ohmura Y, Kurozumi A, Matsushita T, Ohno S, Kanazawa S and Asaumi J: The usefulness of Readout-segmented echo-planar imaging (RESOLVE) for bio-phantom imaging using 3-tesla clinical MRI. Acta Med Okayama 72: 53-59, 2018.

22. Katashima K, Kuroda M, Ashida M, Sasaki T, Taguchi T, Matsuzaki H, Murakami J, Yanagi Y, Hisatomi M, Hara M, et al: In vitro assessment of factors affecting the apparent diffusion coefficient of Jurkat cells using bio-phantoms. Acta Med Okayama 67: 359-367, 2013.

23. Schneider CA, Rasband WS and Eliceiri KW: NIH image to ImageJ: 25 years of image analysis. Nat Methods 9: 671-675, 2012. 
24. Tabesh A, Jensen JH, Ardekani BA and Helpern JA: Estimation of tensors and tensor-derived measures in diffusional kurtosis imaging. Magn Reson Med 65: 823-836, 2011.

25. Stehling MK, Turner R and Mansfield P: Echo-planar imaging: Magnetic resonance imaging in a fraction of a second. Science 254 43-50, 1991.

26. Porter DA and Heidemann RM: High resolution diffusion-weighted imaging using readout-segmented echo-planar imaging, parallel imaging and a two-dimensional navigator-based reacquisition. Magn Reson Med 62: 468-475, 2009.

27. Hempel JM, Schittenhelm J, Bisdas S, Brendle C, Bender B, Bier G, Skardelly M, Tabatabai G, Castaneda Vega S, Ernemann U and Klose U: In vivo assessment of tumor heterogeneity in WHO 2016 glioma grades using diffusion kurtosis imaging: Diagnostic performance and improvement of feasibility in routine clinical practice. J Neuroradiol 45: 32-40, 2018.

28. Qi C, Yang S, Meng L, Chen H, Li Z, Wang S, Jiang T and Li S: Evaluation of cerebral glioma using $3 \mathrm{~T}$ diffusion kurtosis tensor imaging and the relationship between diffusion kurtosis metrics and tumor cellularity. J Int Med Res 45: 1347-1358, 2017.
29. Wu R, Suo ST, Wu LM, Yao QY, Gong HX and Xu JR: Assessment of chemotherapy response in non-Hodgkin lymphoma involving the neck utilizing diffusion kurtosis imaging: A preliminary study. Diagn Interv Radiol 23: 245-249, 2017.

30. Wang F, Jin D, Hua XL, Zhao ZZ, Wu LM, Chen WB, Wu GY, Chen XX and Chen HG: Investigation of diffusion kurtosis imaging for discriminating tumors from inflammatory lesions after treatment for bladder cancer. J Magn Reson Imaging 48: 259-265, 2018.

31. Barrett T, McLean M, Priest AN, Lawrence EM, Patterson AJ, Koo BC, Patterson I, Warren AY, Doble A, Gnanapragasam VJ, et al: Diagnostic evaluation of magnetization transfer and diffusion kurtosis imaging for prostate cancer detection in a re-biopsy population. Eur Radiol 28: 3141-3150, 2018.

32. Hales TC: A proof of the Kepler conjecture. Ann Math 162: 1065-1185, 2005.

33. Einstein A: Investigations on the Theory of the Brownian Movement. Fürth R (ed). Dover Publ. Inc., New York, NY, pp81, 1956. 